\title{
COUPLED AND MIXED COUPLED HYBRID FIXED POINT PRINCIPLES IN A PARTIALLY ORDERED BANACH ALGEBRA AND PBVPS OF NONLINEAR COUPLED QUADRATIC DIFFERENTIAL EQUATIONS
}

\author{
Bapurao C. Dhage
}

\begin{abstract}
In this paper we prove some coupled and mixed coupled hybrid fixed point theorems involving different algebraic combinations of three operators and coupled operators in a partially ordered Banach algebra by an application of a coupled hybrid fixed point principle for partially condensing coupled mappings developed in Dhage [J. Fixed Point Theory Appl. 19 (2017), 2541-2575]. Our approach is based on the partial Kuratowskii measure of noncompactness with maximum property and is somewhat different from the approach of coupled hybrid fixed point theorems presented in Dhage [J. Fixed Point Theory Appl. 19 (2017), 3231-3264]. We apply our newly developed abstract mixed coupled hybrid fixed point theorems along with algorithms to a couple of nonlinear first and second order coupled quadratically perturbed hybrid differential equations with the periodic boundary conditions for proving the existence and approximation theorems under certain mixed hybrid conditions from algebra, analysis and topology. The abstract existence and approximation results of the coupled quadratic periodic boundary value problems of first and second order ordinary differential equations are also illustrated by presenting a few numerical examples. We claim that the results of this paper are new to the literature on nonlinear analysis applications.
\end{abstract}

Mathematics subject classification (2010): 47H07, 47H10, 34A12, 34A45.

Keywords and phrases: Partially ordered Banach algebra, partial measure of noncompactness, Dhage monotone iteration principle, coupled hybrid fixed point theorem, coupled quadratic periodic boundary value problems, existence and approximation theorem.

\section{REFERENCES}

[1] J. APPELL, Measures of noncompactness, condensing operators and fixed points: An applicationoriented survey, Fixed Point Theory 6 (2005), 157-229.

[2] J. Banas, K. Goebel, Measures of Noncompactness in Banach Space, in: Lecture Notes in Pure and Applied Mathematics, Vol. 60, Dekker, New York, 1980.

[3] V. BERINDE, Generalized coupled fixed point theorem for mixed monotone mappings in partially ordered metric spaces, Nonlinear Anal. 74 (2011), 7347-7355.

[4] T. G. Bhaskar, V. LaKshmikantham, Fixed point theorems in partially ordered metric spaces and applications, Nonlinear Analysis: TMA 65 (2006), 1379-1393.

[5] S. CARL, S. HeK KILÄ, Fixed Point Theory in Ordered Sets and Applications, Springer, 2011.

[6] S. S. CHANG, Y. H. MA, Coupled fixed points for mixed monotone condensing operators and an existence theorem of the solutions for a class of functional equations arising in dynamic programming, J. Math. Anal. Appl. 160 (1991), 468-479.

[7] K. Deimling, Nonlinear Functional Analysis, Springer Verlag, 1985.

[8] B. C. Dhage, Some fixed point theorems in ordered Banach spaces and applications, Math. Student 61 (1992), 81-88.

[9] B. C. Dhage, Fixed point theorems in ordered Banach algebras and applications, Pan Amer. Math. J. 9 (4) (1999), 93-102. 
[10] B. C. DhaGe, Remarks on two fixed points theorems involving the sum and the products of two operators, Computers and Math. Appl. 46 (2003), 1779-1785.

[11] B. C. DHAGE, Nonlinear alternatives with applications to nonlinear perturbed differential equations, Nonlinear Studies 13 (2006), 343-354.

[12] B. C. DHAGE, Quadratic perturbations of periodic boundary value problems of second order ordinary differential equations, Differ. Equ. Appl. 2 (2010), 465-486.

[13] B. C. DHAGE, Basic results in the theory of hybrid differential equations with mixed perturbation of second type, Funct. Diff. Equ. 19 (1-2) (2012), 11-29.

[14] B. C. DHAGE, Hybrid fixed point theory in partially ordered normed linear spaces and applications to fractional integral equations, Differ. Equ. Appl. 5 (2013), 155-184.

[15] B. C. Dhage, Partially condensing mappings in ordered normed linear spaces and applications to functional integral equations, Tamkang J. Math. 45 (2014), 397-426.

[16] B. C. DHAGE, Nonlinear D -set-contraction mappings in partially ordered normed linear spaces and applications to functional hybrid integral equations, Malaya J. Matematik 3 (2015), 62-86.

[17] B. C. Dhage, Some generalizations of a hybrid fixed point theorem in a partially ordered metric space and nonlinear functional integral equations, Differ. Equ. Appl. 8 (2016), 77-97.

[18] B. C. DHAGE, Approximating coupled solutions of coupled HPBVPs of nonlinear ordinary differential equations, Kyungpook Math. J. 56 (1) (2016), 221-231.

[19] B. C. DHAGE, Coupled hybrid fixed point theory in a partially ordered metric space and attractivity of nonlinear hybrid fractional integral equations, J. Fixed Point Theory Appl. 19 (2017), 2541-2575.

[20] B. C. DHAGE, Coupled hybrid fixed point theory involving the sum and product of three coupled operators in a partially ordered Banach algebra with applications, J. Fixed Point Theory Appl. 19 (2017), 3231-3264.

[21] B. C. DHAGE, Dhage iteration method for PBVPs of nonlinear first order hybrid integro-differential equations, Int. J. Nonlinear Anal. Appl. 8 (2017), 95-112.

[22] B. C. DHAGE, Dhage iteration method in the theory of ordinary nonlinear PBVPs of first order functional differential equations, Commun. Optim. Theory 2017 (2017), Article ID 32, pp. 22.

[23] B. C. DHAGE, A coupled hybrid fixed point theorem involving the sum of two coupled operators in a partially ordered Banach space with applications, Differ. Equ. Appl. 9 (4) (2017), 453-477.

[24] B. C. Dhage, Some variants of two basic hybrid fixed point theorems of Krasnoselskii and Dhage with applications, Nonlinear Studies 25 (3) (2018), 559-573.

[25] B. C. DHAGE, A coupled hybrid fixed point theorem for sum of two mixed monotone coupled operators in a partially ordered Banach space with applications, Tamkang J. Math. 50 (1) (2019), 1-36.

[26] B. C. DHAGE, S. B. DHAGE, Approximating solutions of nonlinear first order ordinary differential equations, GJMS Special Issue for Recent Advances in Mathematical Sciences and Applications 13, GJMS Vol. 2, No. 2, (2014), 25-35.

[27] B. C. Dhage, S. B. Dhage, Coupled hybrid fixed point theorem in partially ordered metric spaces with applications, Nonlinear Studies 21 (4) (2014), 675-656.

[28] B. C. Dhage, S. B. Dhage, Approximating positive solutions of PBVPs of nonlinear first order ordinary hybrid differential equations, Appl. Math. Lett. 46 (2015), 133-142.

[29] B. C. DhAGE, S. HeIKKILÄ, On nonlinear boundary value problems with deviating arguments and discontinuous right hand side, J. Appl. Math. Stochastic Anal. 6 (1) (1993), 83-92.

[30] D. Guo, V. Lakshmikantham, Nonlinear Problems in Abstract Cones, Academic Press, New York, London 1988.

[31] S. Heik Kil Ä, V. Lakshmikantham, Monotone Iterative Techniques for Discontinuous Nonlinear Differential Equations, Marcel Dekker inc., New York 1994.

[32] J. J. NiETo, Basic theory for nonresonance impulsive periodic problems of first order, J. Math. Anal. Appl. 205 (1997), 423-433.

[33] J. J. Nieto, Nonlinear second order periodic value problems with Carathéodory functions, Appl. Anal. 34 (1989), 111-128.

[34] J. J. Nieto, R. RodRigueZ-Lopez, Existence and approximation of solution for nonlinear differential equations with periodic boundary conditions, Comput. Math. Appl. 40 (2000), 435-442.

[35] J. J. Nieto AND R. RodrigueZ-LopeZ, Contractive mappings theorems in partially ordered sets and applications to ordinary differential equations, Order 22 (2005), 223-239.

[36] P. Nistri, V. V. OBukhovski, P. ZECCA, On the solvability of systems of inclusions involving noncompact operators, Trans. Amer. Math. Soc. 342 (1994), no. 2, 543-562. 
[37] M. H. Protter, H. F. Weinberger, Maximum Principles in Differential Equations, Prentice-Hall, Englewood Cliffs, N. J. 1967.

[38] Y. Sun, A fixed point theorem for mixed monotone operator with applications, J. Math. Anal. Appl. 156 (1991), 240-252.

[39] H. YAng, R. P. Agarwal, H. K. NAShine, Y. LiAng, Fixed point theorems in partially ordered Banach spaces with applications to nonlinear fractional evolution equations, J. Fixed Point Theory Appl. 19 (2017), 1661-1678.

[40] E. ZeIdLer, Nonlinear Functional Analysis and Its Applications: Part.I, Springer-Verlag, New York, 1985. 